\title{
La calidad como valor añadido determinante en el consumo de contenidos informativos de pago en internet
}

\section{Quality as an added value in the paid-for information content on internet}

\author{
Ana Segura-Anaya; Carmen Marta-Lazo; Antonia-Isabel Nogales-Bocio
}

Cómo citar este artículo:

Segura-Anaya, Ana; Marta-Lazo, Carmen; Nogales-Bocio, Antonia-Isabel (2019). "La calidad como valor añadido determinante en el consumo de contenidos informativos de pago en internet". El profesional de la información, v. 28, n. 6, e280607.

https://doi.org/10.3145/epi.2019.nov.07

Artículo recibido el 10-06-2019 Aceptación definitiva: 25-10-2019

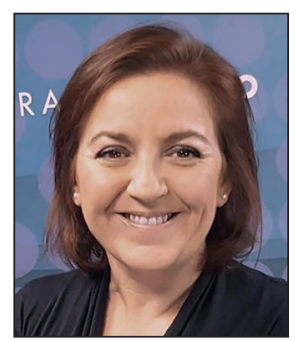

Ana Segura-Anaya $\bowtie$ https://orcid.org/0000-0003-0332-9445

Universidad de Zaragoza Facultad de Filosofía y Letras San Juan Bosco, 7 50009 Zaragoza, España asegura@unizar.es

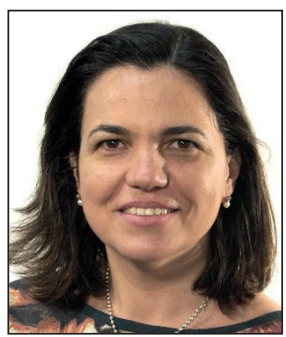

Carmen Marta-Lazo https://orcid.org/0000-0002-0004-1094

Universidad de Zaragoza Facultad de Filosofía y Letras

San Juan Bosco, 7 50009 Zaragoza, España cmarta@unizar.es

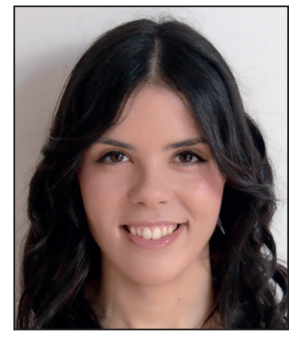

\section{Antonia-Isabel Nogales-Bocio} https://orcid.org/0000-0003-0050-6979

Universidad de Zaragoza

Facultad de Filosofía y Letras

San Juan Bosco, 7

50009 Zaragoza, España

anogales@unizar.es

\section{Resumen}

El cobro por los contenidos informativos es una de las principales preocupaciones para los medios de comunicación que buscan un modelo de negocio en internet. La percepción del "gratis total" con la que muchos usuarios se acercan a la Red, la caída de ingresos por publicidad y la irrupción en el mercado de nuevos agentes como las redes sociales obligan a reflexionar sobre el valor añadido que será determinante en la disposición al pago por contenidos. La presente investigación analiza el valor de la calidad desde dos puntos de vista y con dos metodologías complementarias, la de los profesionales que gestionan empresas informativas y la de los usuarios que consumen los contenidos. Se da respuesta a qué se entiende por calidad y si este elemento es suficiente para determinar la intención de pago. Como principal conclusión llegamos a determinar que la calidad es el principal valor, que tanto los profesionales como los usuarios destacan por encima de todos los demás y que se asocia a valores como la exclusividad, diferenciación, especialización o accesibilidad.

\section{Palabras clave}

Internet; Pago por contenidos; Información; Calidad; Modelo de negocio; Poder; Medios de comunicación; Medios digitales; Cibermedios; Periodismo digital; Consumo de información; Audiencias; Estudios de usuarios; Entrevistas; Encuestas. 


\begin{abstract}
Paid-for information content has become a major concern among media looking for a suitable business model on the internet. The "totally-free" perception presumed by a significant number of users, the sharp drop in advertising revenues and the entry into the market of new agents such as social media, demand a reflection about that added value which shall prove decisive to foster an inclination towards paid content. The present research work analyses the value of quality from two points of view and through two complementary methods: those of the professionals managing media corporates, on the one hand, and those of users consuming content, on the other. Hence, answers are provided to questions raised, concerning the definition and perception of quality, and whether that sole element is enough to prompt the intention to pay. As an overall conclusion, it is finally determined that quality is indeed the primary value, as stressed by both professional and users, above everything else, and it is associated to values such as exclusiveness, differentiation, specialisation and accessibility.
\end{abstract}

\title{
Keywords
}

Internet; Paid-content; Information; Quality; Business models; Power; Mass media; Cybermedia; Digital media; Digital journalism; Consumption of information; Audiences; Users studies; Interviews; Surveys.

\section{Introducción}

En la era de internet los consumidores, convertidos en productores de contenido o prosumidores, tienen a su disposición una oferta de contenidos informativos infinita, que se renueva constantemente y para la que no existen cierres de edición ni días festivos. Son contenidos tanto globales como de nicho o altamente especializados, a los que en gran medida se accede gratuitamente, donde, cuando y como se quiere, gracias a los soportes de movilidad, que cada vez son más accesibles y cómodos. Pero también proliferan las noticias falsas y sin contrastar, la información basura o los enlaces trampa que sólo buscan incrementar el número de visitas a una web. El mismo consumidor que antes era, salvo excepciones, agente pasivo receptor del mensaje, es ahora parte activa que comenta, publica, comparte (Iniesta-Alemán; Marta-Lazo; Zaro-Becas, 2018) y en ocasiones incluso condiciona los temas y su tratamiento como nunca antes lo había hecho, a través de la acción directa en foros y redes sociales, pero también a través de los datos que muchas veces inconscientemente va dejando en su propio consumo en internet (Lastra, 2016; Aparici; García-Marín, 2018).

El ecosistema de la comunicación (Campos-Freire, 2010a) está en pleno proceso de cambio y desde finales del siglo XX los medios viven una situación de crisis económica y sistémica (Reig, 2015) que comenzó afectando a la prensa en papel pero que poco a poco está obligando a replantear su modelo tanto a la radio como a la televisión. El escenario actual anuncia el fin del modelo tal y como lo conocíamos y augura un futuro, que ya es presente, en internet. Las empresas periodísticas se enfrentan a la caída de los ingresos por publicidad (Cabezuelo-Lorenzo, 2013), la necesidad de rejuvenecer, renovar y digitalizar las redacciones, la propia reconversión tecnológica de los procesos de producción de la información (Marta-Lazo; Segura-Anaya; Martínez-Oliván, 2017) y la competencia de las plataformas y redes sociales que distribuyen contenidos informativos, muchas veces procedentes de fuentes que no reciben contraprestación económica.

En esta investigación tratamos de responder a las siguientes preguntas:

- ¿Pueden confluir en este escenario los intereses de los medios y de los consumidores?

- ¿Es posible construir un nuevo modelo viable económicamente, que ofrezca contenidos de calidad a un usuario interesado y participativo?

Nuestro trabajo analiza el reto desde dos perspectivas: la de los profesionales de los medios y la de los usuarios. El principal objetivo es analizar e intentar determinar la relevancia que posee el concepto "calidad" y las implicaciones que tiene para ambos grupos.

\section{Estado de la cuestión}

La revolución que internet ha supuesto en el mundo de la comunicación va mucho más allá de la mera distribución de contenidos. Alcanza a los propios contenidos, a las empresas periodísticas, a las nuevas compañías de distribución surgidas de internet, a los consumidores y, en general, a las relaciones que se establecen entre todos ellos. Los medios han tenido que agruparse en corporaciones o grupos multimedia para poder sobrevivir. Las redacciones de estos medios han incorporado nuevos perfiles profesionales vinculados al desarrollo y uso de la tecnología. Los redactores y editores han necesitado adquirir nuevas competencias para asumir tareas propias de la producción de contenidos adaptados a los soportes y formatos de distribución de la información. Por su parte, la propia información ha roto los límites que hasta ahora se asociaban a cada medio para hacer convivir texto, imagen y vídeo y abrirse a nuevas e infinitas posibilidades tecnológicas, mientras las redes sociales y las plataformas de distribución de contenidos irrumpían en el escenario como nueva opción informativa, no siempre con garantías. Finalmente, la facilidad para producir, publicar y distribuir contenidos informativos en internet ha cuestionado la figura del editor como responsable de priorizar los temas, supervisor de la línea editorial y garantía de veracidad. En este contexto, el consumidor cobra un protagonismo muy superior al que había tenido en etapas previas. 


\subsection{El prosumidor y los patrones de consumo}

Toffler (1981), McLuhan y Nevitt (1972), Levine y Locke (2000), Jenkins (2008) y Gubbins (2012) analizan el concepto de prosumidor y el cambio de actitud de los usuarios, al intervenir en el proceso de la comunicación de forma activa, mientras Jenkins (2006) define como cultura de la convergencia el escenario social en el que estos usuarios activos crean, difunden y comparten.

Según datos de la Comisión Europea, el 71,6\% de los usuarios de internet se conectan diariamente y lo hacen mayoritariamente para usar aplicaciones $(67,1 \%)$, leer prensa digital $(66,2 \%)$ y entrar en redes sociales $(60,1 \%)($ Ontsi, $2017 a)$. Casi la mitad de los españoles (48,5\%) lee noticias en internet a diario y un 33\% escucha música o programas en formato podcast o streaming. La televisión de pago, consolidada en EUA y los países del norte de Europa, sigue en España su ascenso imparable y certifica, según la Comisión Nacional de los Mercados y la Competencia (2017), la mayor cifra por facturación en toda la serie histórica, lo que le lleva a superar por primera vez en nuestro país los ingresos por publicidad de la televisión en abierto, sin incluir las subvenciones de instituciones públicas. Además, según el citado Ontsi (Observatorio Nacional de las Telecomunicaciones) (2017b) al terminar 2017, por primera vez en España los ordenadores lograron desplazar a la televisión, con un $84,9 \%$ de presencia en los hogares frente al 84,3\% de receptores.

Mientras el consumo crece imparable, en la Red se ha consolidado una cultura de la gratuidad, en la que el acceso a la información de actualidad se realiza sin efectuar ningún pago y se genera una resistencia cada vez mayor a éste entre los usuarios (Miguel-De-Bustos; Casado-Del-Río, 2010; MacNamara, 2010; Castells, 2009; Chyi; Yang, 2009). El informe Digital news report (Reuters Institute, 2017) desvela respecto al del año anterior una caída del 7\% (del 18\% al 11\%) entre los internautas que muestran disposición a pagar por contenidos informativos en un futuro, y se mantienes estancados en un $9 \%$ los que afirman hacerlo. El mismo informe establece que el perfil del comprador de noticias digitales es un varón joven, de entre 25 y 34 años, con un alto nivel de ingresos y estudios, que acude con mucha frecuencia a los medios de comunicación, digitales y tradicionales para satisfacer su elevado interés en la actualidad. Entre los motivos que alega para el pago están la posibilidad de acceder a las noticias desde el móvil con una variedad de fuentes, y el haber accedido a través de una oferta de suscripción, la modalidad preferida.

\subsection{Calidad como valor añadido}

Ante este escenario surge la siguiente pregunta: ¿cuáles son los contenidos por los que los usuarios estarían dispuestos a pagar? Parece que los contenidos audiovisuales, breves, dinámicos, fácilmente accesibles, comprensibles y compartibles ganan frente a la información tratada de forma extensa y en profundidad. Gallardo-Olmedo (2011) defiende que frente a la lectura pausada de la prensa en papel, los lectores en internet ya no consumen periódicos sino noticias. Vara-Miguel, Sanjurjo y Díaz-Espina (2012) destacan que los medios tradicionales compiten con proveedores de contenidos que basan su modelo económico en la cantidad, en el volumen de usuarios que la visitan sin importar la calidad del contenido ofrecido.

En un contexto como el que nos ocupa, con una oferta ilimitada para el internauta, cobra especial relevancia el concepto de valor percibido por los consumidores, al que nos acercamos desde la perspectiva del marketing relacional. Kotler (1996), Zeithaml (1988), Woodruff (1997), y Gallarza y Gil-Saura (2006) definen y analizan la naturaleza y dimensiones del concepto de valor añadido, su carácter dinámico y la vinculación con las percepciones de los consumidores (valor subjetivo), porque está basado en percepciones individuales (Woodruff, 1997; Parasuraman, 1997). La mayoría de las investigaciones que analizan los antecedentes del valor añadido hacen referencia a la calidad, el precio y la experiencia de compra o consumo (Parasuraman; Grewal, 2000; Kerin; Varadarajan; Peterson, 1992) y destacan tres factores clave en el valor añadido percibido: la calidad del producto, el precio y la calidad de los servicios adicionales.

La American Society for Quality define la calidad como:

"la totalidad de funciones y características de un producto que permiten satisfacer una determinada necesidad" (Gutiérrez-Coba, 2006).

En el caso del periodismo, la calidad se relaciona también con la vocación (y la percepción) de servicio público del medio (Echeverría; González, 2018), así como con la capacidad de superar los condicionantes mercantiles, pues las preocupaciones del mercado determinan en muchas ocasiones el contenido en la industria mediática (Picard, 2004). La percepción de la calidad que tienen los usuarios de prensa influye en la credibilidad y en el valor de las marcas periodísticas (Victoria-Mas; Lacasa-Mas, 2016). Estos autores distinguen por ejemplo entre "calidad técnica" y "calidad literaria" para medir el valor de marca en una publicación periódica.

La calidad y la credibilidad, junto a la reputación y la transparencia, son las bases sobre las que se fortalece la confianza del usuario (Campos-Freire, 2010b). De hecho la información cobra valor a medida que se profesionaliza, por lo que la mediación profesional, la función del gatekeeper, sería más necesaria que nunca en los tiempos de la infoxicación.

\subsection{Valor añadido y pago por contenidos}

Holbrook y Schindler (1994) ponen de relieve la experiencia de consumo como determinante en la percepción del valor añadido. La oportunidad que para la compra online ofrece internet otorga una nueva perspectiva al concepto de valor 
añadido percibido. Chang, Cheung y Lai (2005) destacan que las ventajas adicionales se consideran como uno de los principales factores antecedentes de la compra online. Chen y Dubinsky (2003) desarrollan un modelo de análisis de la generación de valor para los consumidores en el ámbito del comercio electrónico con cuatro componentes: la experiencia de compra online, la calidad percibida del producto, el riesgo percibido, y el precio.

La teoría de la equidad (Oliver; Swan, 1989a; 1989b) se aplica al consumo entendiéndola como comparaciones entre datos de entrada y datos de salida. Los consumidores evaluamos en cada decisión de consumo la inversión y el beneficio que nos supone y tomamos la decisión de comprar cuando este balance nos es favorable. Sin embargo, en la Red se ha consolidado una cultura de la gratuidad, en la que los usuarios acceden a los contenidos sin pagar y este hábito genera una resistencia cada vez mayor al propio pago (Miguel-De-Bustos; Casado-del-Río, 2010; MacNamara, 2010; Castells, 2009; Chyi; Yang, 2009).

En líneas generales, el valor añadido es para la gran mayoría de los autores (Herbert; Thurman, 2007; Parra et al., 2008; Franco-Álvarez, 2005; Gallardo-Olmedo, 2011; Goyanes, 2013a) la clave en la predisposición al pago por los contenidos informativos. Sólo si los medios digitales son capaces de aportar ese valor añadido, los internautas estarán dispuestos a pagar por los contenidos digitales. La clave es determinar las variables que constituyen ese extra por el que los consumidores piensan que merece la pena pagar, y es que la información por sí misma ya no es atractiva en cuanto puede conseguirse de distintas fuentes, muchas de ellas gratuitas aun a costa de sobrecargas publicitarias o noticias sin contrastar.

Los principales estudios sobre pago por contenidos (WAN, 2010; Pew Research Center, 2010; PWC, 2014; 2017; Reuters Institute, $2014 ; 2015 ; 2016$; 2017) cifran las personas dispuestas a pagar por consumir noticias online entre el $10 \%$ y $20 \%$ (los más optimistas) del total de lectores. El arraigo de la gratuidad es tal que el $74 \%$ de los usuarios buscaría otro medio de acceso libre si su sitio web favorito decidiera cobrar por las noticias (WAN, 2010). Esta tendencia se ha visto reforzada por la web 2.0, cuya lógica se basa en la colaboración y la cooperación y el desarrollo de las redes sociales que permiten acceder a contenidos directos mediante enlaces (Marta-Lazo; García-Idiakez, 2014).

Diversos factores influyen en la decisión del usuario de pagar por contenidos (Chyi, 2012), y el carácter de versión digital de los cibermedios puede potenciar o restar en el valor asociado a las noticias (Ha; Zhang, 2017). En líneas generales, para crear un mayor valor los medios digitales -tanto de pago como gratuitos-suelen ofrecer información que por algún motivo tiene un valor añadido, y también aprovechan el valor de marca de las ediciones impresas (Goyanes, 2013b). En el sector de la información en internet, es el propio canal el que ofrece un valor añadido frente al medio impreso tradicional para los lectores de periódicos (Chyi; Lasorsa, 2002; De-Waal; Schoenbach, 2010) como la inmediatez, la posibilidad de acudir a diferentes fuentes de información de una manera sencilla y sin apenas esfuerzo, o la actualización constante de las noticias. Casero-Ripollés (2012) destaca que los valores más relevantes en los medios informativos digitales para la audiencia más joven, comprendida entre 15 y 30 años, son actualización, multimedialidad, personalización, relevancia, y participación.

Wang et al. (2005) establecen que la disposición de un usuario a pagar por el contenido o los servicios online está positivamente relacionada con su percepción de la comodidad, la esencialidad, el valor añadido y la calidad del servicio, así como a su tasa de uso de un determina-

do servicio. Además, su disposición a pagar se relaciona negativamente con la percepción de injusticia en un modelo de servicio online de suscripción. Los datos planteados por Goyanes y Vara-Miguel (2017) muestran que aquellos que desconfían de las noticias tienen más probabilidad de pagar por información digital, dado que los lectores sólo pagan por aquello que consideran deseable. Por otro lado, los jóvenes que crecieron con internet parecen estar más dispuestos a pagar por el contenido online (Yang et al., 2015). Pese a todo y a los muchos esfuerzos para aumentar el cobro por contenido, los ingresos por publicidad siguen dominando la cuenta de resultados de los medios de comunicación digitales (Cea-Esteruelas, 2013).

Por lo tanto, teniendo en cuenta el contexto de partida y el estado de la cuestión, planteamos las siguientes preguntas de investigación:

PI1: ¿Qué valor tiene la calidad de la información para los agentes implicados en el proceso: medios y prosumidores? PI2: ¿En qué reside la motivación del consumidor para llegar a pagar por contenidos informativos?

PI3: ¿Cómo se relacionan las variables demográficas estudiadas con la disposición al pago?

PI4: ¿Es la calidad ese factor determinante que condiciona la disposición al pago por contenidos informativos?

\section{Metodología}

Analizamos el valor que la calidad de la información tiene para los dos agentes directamente implicados en el proceso de la comunicación, los medios y los prosumidores, a través de la triangulación con dos metodologías complementa- 
rias, cualitativa y cuantitativa. Por una parte, mediante entrevistas individuales semiestructuradas a distintos perfiles profesionales en medios de comunicación y empresas informativas y, por otra, a través de una encuesta a una muestra representativa de 764 consumidores de contenidos informativos en internet.

Respecto a las entrevistas en profundidad, se han realizado a un panel de siete expertos en distintas áreas profesionales (gestión empresarial, contenidos, marketing, desarrollo de producto) y medios de comunicación (prensa, radio, televisión, agencias de noticias), dado que nuestra intención es ofrecer una visión panorámica global y lo más amplia posible de la situación de un mercado muy complejo y en pleno proceso de transformación.

La muestra se compone de siete entrevistas semiestructuradas a profesionales en activo del mundo de la comunicación, el marketing y la prensa digital. Hemos considerado interesante ir más allá de los directores de los medios e incorporar otros perfiles profesionales que pudieran aportar una visión lo más amplia posible del proceso de la comunicación digital, más allá de los meros contenidos. Entendemos que el problema de la monetización de contenidos en internet y el desarrollo de un modelo de negocio trasciende a un área determinada de cada organización e implica necesariamente a productores de contenidos tanto en prensa, radio o televisión, gestores, responsables de marketing, expertos comerciales, desarrolladores de sistemas, etc.

Se trató de elegir entrevistados de reconocido prestigio en su campo profesional, que hubieran realizado aportaciones relevantes (bien por haber sido pioneros, por su trayectoria profesional o por su profundo conocimiento del medio), que ofrecieran una mayor oportunidad de aprendizaje y una visión global y lo más profunda posible de problema analizado. Los perfiles, reseñados en la tabla 1, son los siguientes:

- Perfil 1. Directivos de medios de comunicación (prensa, radio o TV) con experiencia en desarrollos digitales, que hayan participado activamente en la creación de nuevas propuestas de comunicación digital o en la transformación de medios convencionales a digitales.

- Perfil 2. Directivos de asociaciones profesionales vinculadas a la comunicación digital, como AERO (Asociación Española de Radio Online), Online Publishers Association Europe, Agrupación de Medios Digitales de España, Aeepp (Asociación Española de Publicaciones Periódicas), etc.

- Perfil 3. Periodistas free-lance, autónomos y emprendedores que hayan creado en internet un medio digital como iniciativa de autoempleo. Según los datos de la Asociación de la Prensa de Madrid en su Libro blanco de la prensa 2015, desde el 1 de enero de 2008 al 25 de mayo de 2015, se crearon en España 451 medios digitales.

- Perfil 4. Expertos en marketing que aporten una visión puramente económica del problema que planteamos.

Tabla 1. Panel de profesionales entrevistados

\begin{tabular}{|c|c|c|c|c|c|}
\hline Nombre & Sector & Empresa o entidad & Puesto & Lugar & Duración \\
\hline Entrevistado 1 & $\begin{array}{l}\text { Perfil } 2 \\
\text { Radio digital }\end{array}$ & $\begin{array}{l}\text { Aero (Asociación Española } \\
\text { de Radio Online) }\end{array}$ & $\begin{array}{l}\text { Miembro de la junta directiva. Profesional con } \\
\text { más de } 30 \text { años de experiencia en radio. }\end{array}$ & Madrid & $41^{\prime} 52^{\prime \prime}$ \\
\hline Entrevistado 2 & $\begin{array}{l}\text { Perfil } 3 \\
\text { Prensa digital }\end{array}$ & $\begin{array}{l}\text { Administración Pública. } \\
\text { Gobierno de Aragón }\end{array}$ & $\begin{array}{l}\text { Periodista. Cargo público en ámbito tecnológi- } \\
\text { co. Experiencia como emprendedor }\end{array}$ & Zaragoza & $32^{\prime}$ \\
\hline Entrevistado 3 & $\begin{array}{l}\text { Perfil } 4 \\
\text { Marketing }\end{array}$ & $\begin{array}{l}\text { Factor I+D Consultoría en } \\
\text { marketing digital }\end{array}$ & $\begin{array}{l}\text { Experto en marketing digital. Consultor exter- } \\
\text { no en empresas de primer nivel }\end{array}$ & Zaragoza & $34^{\prime} 41^{\prime \prime}$ \\
\hline Entrevistado 4 & $\begin{array}{l}\text { Perfil } 1 \\
\text { Televisión }\end{array}$ & Arait Multimedia S.A. & $\begin{array}{l}\text { Experto en televisión con amplia experien- } \\
\text { cia en desarrollo de producción y nuevos } \\
\text { formatos }\end{array}$ & Madrid & $16^{\prime} 33^{\prime \prime}$ \\
\hline Entrevistado 5 & $\begin{array}{l}\text { Perfil } 1 \\
\text { Grupo multimedia }\end{array}$ & $\begin{array}{l}\text { logenia Digital (Joint } \\
\text { vernture de Grupo Heraldo } \\
\text { y Grupo la Información) }\end{array}$ & $\begin{array}{l}\text { Directivo en grupo de comunicación con } \\
\text { importante crecimiento nacional }\end{array}$ & Zaragoza & $21^{\prime} 42^{\prime \prime}$ \\
\hline Entrevistado 6 & $\begin{array}{l}\text { Perfil } 3 \\
\text { Prensa digital }\end{array}$ & Grupo Aragón Digital & $\begin{array}{l}\text { Periodista. Directivo y socio fundador en } \\
\text { proyecto digital pionero en los } 90\end{array}$ & Zaragoza & $30^{\prime} 43^{\prime \prime}$ \\
\hline Entrevistado 7 & $\begin{array}{l}\text { Perfil } 2 \\
\text { Consultoría }\end{array}$ & $\begin{array}{l}\text { Online Publishers Associa- } \\
\text { tion Europe (OPA Europe) }\end{array}$ & $\begin{array}{l}\text { Directivo en Online Publishers Association } \\
\text { Europe }\end{array}$ & Barcelona & $30 \prime 09^{\prime \prime}$ \\
\hline
\end{tabular}

Con las entrevistas se pretendió definir una serie de variables que los profesionales de los medios considerasen determinantes a la hora de dibujar un modelo de negocio y favorecer la intención de pago por contenidos por parte de los lectores o receptores. Esas variables serían clave para diseñar la segunda parte de nuestro análisis, centrado en este caso en el punto de vista del consumidor.

Las impresiones y las conclusiones o variables que extraemos de las entrevistas en profundidad han servido para diseñar la encuesta (segunda parte de nuestro análisis), que se ha realizado a una muestra aleatoria no probabilística de usuarios de internet, de acuerdo a variables extraídas de los datos del CIS (edad, sexo, nivel educativo y nivel de ingresos) como representativos de la población española y con un índice de confianza del 95\%. La encuesta se ha realizado a un total de 764 usuarios de internet, con un porcentaje del 41,37\% de hombres y el 58,63\% de mujeres. Por franjas de edad se ha intentado reproducir la pirámide poblacional que refleja la sociedad española, estableciendo franjas de edad de 14 a 
65 y más años. Del mismo modo, se ha contemplado que más de la mitad de la muestra sean residentes en entornos claramente urbanos, entendiendo como tales localidades mayores de 50.000 habitantes. El 10\% de los encuestados reside en ciudades de entre 50.000 y 200.000 habitantes y el $51 \%$ en ciudades de más de 200.000 habitantes, cifras muy próximas a los datos del censo publicados por el Instituto Nacional de Estadística para el año 2017.

Hemos considerado determinante introducir la variable educación en nuestro estudio y discriminar entre estudiantes, empleados, funcionarios, autónomos, desempleados y jubilados, con la representación que se muestra en el gráfico 1.

Hemos considerado relevante introducir la variable nivel de estudios y nivel de ingresos, con una representación que puede observarse en el gráfico 2, porque entendemos que ambas pueden ser determinantes en la percepción de los consumidores. Cabe recordar que algunos de los códigos definidos y utilizados en la segunda fase, de entrevistas semiestructuradas a profesionales de la comunicación como la exclusividad, la calidad y la especialización, pueden relacionarse directamente con variables como el nivel de estudios o de ingresos, relación que comprobaremos a lo largo del estudio.

La muestra es por lo tanto representativa de la sociedad española de acuerdo a los datos de población publicados por organismos oficiales como el Instituto Nacional de Estadística. Esta investigación cuantitativa nos permite así conocer la percepción de los usuarios sobre las variables determinantes en el modelo de la comunicación digital apuntadas por los expertos en un contexto de contaminación informativa, auge de las noticias falsas o depreciación del valor de la información, con las consecuencias que esto puede tener en la sociedad del futuro.

\section{Resultados}

El análisis de los factores subrayados por los entrevistados nos permite proponer cinco variables o conceptos clave que sintetizan los principales factores determinantes del valor añadido percibido por los usuarios en la compra de contenidos informativos en internet. Estas variables se relacionan en un modelo, como muestra el gráfico 4, en el que el valor añadido percibido se fundamenta en la calidad del producto, determinada a su vez por cuatro factores: especialización, diferenciación, exclusividad y accesibilidad.

Por especialización se entiende una oferta dirigida a un mercado de nicho, con consumidores muy motivados y dispuestos a sufragar el coste de un producto de máximo nivel y muy segmentado, en ocasiones debido a una necesidad laboral. Un acceso sencillo, rápido, en multiplataforma y que se adapte a la rápida evolución de los propios dispositivos se apunta como clave en la percepción de un servicio de calidad.

La accesibilidad, que tiene en el desarrollo tecnológico su mejor aliado, se enfrenta sin embargo a los problemas derivados de la propia estructura del mercado, en el que conviven empresas que desarrollan sus propias plataformas de distribución limitando el acceso y las posibilidades de compartir contenidos con otras como estrategia para apuntalar su crecimiento y protegerse de la competencia. 
La diferenciación la otorga la calidad del propio contenido informativo, un contenido propio en un mercado en el que la gran mayoría de medios se limita a replicar comunicados y notas de prensa. Si bien los profesionales entrevistados coinciden en destacar la imposibilidad de seguir apostando por el concepto "exclusiva" en un medio en el que lo nuevo se comparte y copia con regularidad, sí valoran el atractivo de contenidos refrendados por firmas de prestigio $y$ apuestan por los temas propios, informes que complementen la actualidad que es común para todos o las crónicas de autor en el lugar de los hechos.

En el caso de la información digital la exclusividad viene dada por la pertenencia a un grupo diferenciado con el que el usuario se identifica porque comparte sus valores y que trasciende a la figura del mero consumidor. De esta forma, algunos medios convierten a los suscriptores en socios de un proyecto que apoya económicamente un modelo de periodismo con valores concretos, bien la proximidad ideológica o simplemente el interés por un tema concreto de nicho.

La calidad como principal valor añadido, sustentado en las cuatro variables sintetizadas de las entrevistas en profundidad (Marta-Lazo; Segura-Anaya; Martínez-Oliván, 2017), se confronta ahora con las percepciones de los consumidores de información en internet a través de una encuesta que nos permite comparar las conclusiones extraídas de la investigación cualitativa sobre las variables determinantes del pago por contenidos informativos y la relevancia de la calidad en el proceso.

La encuesta realizada a 764 usuarios de internet muestra que los contenidos informativos que los usuarios buscan son contenidos de actualidad, seguidos de la información cultural y de ocio, política nacional e información local y de proximidad.

El perfil de usuario de internet que dibujan los resultados ratifica que el consumo de información es uno de los principales usos e intereses, con el $84,9 \%$ de los encuestados que se define como consumidor habitual. Los más jóvenes demandan más información, no existen grandes diferencias entre hombres y mujeres ni entre los distintos niveles de ingresos y la extensión casi universal de la red hace que tampoco se observen grandes diferencias entre los ámbitos rural y urbano. Sí aparecen como variables determinantes la ocupación (vinculada a la edad, en cuanto los estudiantes son los que más información consumen en internet y los jubilados los que menos) y el nivel de estudios, con mayores consumos entre los que cuentan con estudios superiores y una correspondencia entre bajo nivel de estudios y bajo consumo informativo en internet. Y en un contexto de oferta prácticamente infinita, ¿qué determina la elección de una u otra web para buscar información? Un $67 \%$ de los encuestados priorizan la veracidad de la información y la calidad de los contenidos. Mucho más lejos, casi un $18 \%$ valoran que la página cargue rápido, sin errores y en cualquier navegador. Que la publicidad no sea invasiva sólo es determinante para el 4,7\% de los encuestados y aún menos relevante resulta la usabilidad, los contenidos extra o la adaptación de la interfaz a cualquier dispositivo. La calidad de los contenidos sobresale como la variable más destacada a la hora de elegir una fuente de información en internet, sin diferencias por edad, sexo, educación o ingresos.

La calidad es también la principal motivación que alegan los encuestados para pagar por contenidos informativos, con un $48 \%$ de respuestas coincidentes, seguido por la imposibilidad de encontrar gratis los contenidos y la supresión de la publicidad. Un sentido utilitarista vinculado al trabajo es una de las causas que apuntan hasta el $22,6 \%$ de los encuestados, mientras que la confianza en la veracidad de las informaciones de un medio concreto no llega a alcanzar el $20 \%$. El precio sólo es considerado un factor determinante por el 15,8\% de los encuestados.

Por debajo del 15\% aparecen motivaciones como la proximidad, diferenciación o accesibilidad, todas ellas con un porcentaje de respuestas muy escaso.

En cuanto a diferencias en la variable sexo, los hombres destacan la calidad de los contenidos (52,4\%) frente al grupo de las mujeres, quienes, aunque sigue siendo la opción más elegida, desciende al 44,8\%. La imposibilidad de encontrar 
esos contenidos de forma gratuita en internet $(37,3 \%)$ es la segunda opción con muy poca diferencia por género. La línea editorial del medio marca también una interesante diferencia entre hombres y mujeres, ya que frente al $14,3 \%$ de hombres que eligen la opción, sólo son el $8,7 \%$ de las mujeres. Las mujeres, además de la calidad y la imposibilidad de acceder gratuitamente, priorizan los contenidos extras que puedan ofrecer, un $18,29 \%$ y cuatro puntos porcentuales más que los hombres y los contenidos interesantes para el trabajo, 25,3\% frente al 18,8\%. La sencillez en el acceso, a pesar del escaso porcentaje global, interesa más a las mujeres que a los hombres.

El rechazo al pago por parte de los usuarios encuestados es patente por ejemplo cuando se plantean distintas opciones en el caso de que la web preferida para buscar información decidiera cobrar por los contenidos: un 58,7\% se niega a pagar y está dispuesto a buscar la información en otra fuente.

Si analizamos las motivaciones por franja de edad, la calidad del contenido es la variable más destacada en cifras globales y en porcentajes por todas las franjas de edad, excepto por la de los mayores de 65 de los que apenas un $8 \%$ la eligen. La imposibilidad de encontrar gratis los contenidos es determinante para más del 50\% de los menos de 24 años, pero afecta a apenas el $20 \%$ de los mayores de 65 . En tercer lugar, en cifras globales aparece la posibilidad de acceder a contenidos sin publicidad, algo especialmente valorado por lo más jóvenes y los comprendidos en la franja entre 55 y 65 años. Respecto a los motivos alegados para no pagar por contenidos informativos en internet, es significativo que más del 56\% de los mayores de 65 años encuestados consideren que en internet los contenidos deben ser gratis, frente al 28,8\% de los comprendidos en la franja entre 14 y 24 años. En el extremo opuesto, el $56 \%$ de estos últimos señalan la posibilidad de buscar en otras páginas gratuitas el mismo contenido, una opción que sólo apunta el $25 \%$ de los mayores.

La falta de interés la apuntan el $44 \%$ de los más jóvenes, que también destacan que les parece suficiente la información que reciben de redes sociales, que prefieren la publicidad a pagar por contenidos y que además el sistema de pago les parece poco fiable. El precio es también determinante para uno de cada cuatro encuestados de entre 14 y 34 años.

En la misma línea, si analizamos los datos en función de la ocupación, podemos comprobar que los estudiantes prefieren buscar los contenidos en páginas gratuitas y que en general no los encuentran lo suficientemente interesantes para pagar por ellos. Esta opción es también la más elegida por los autónomos; sin embargo, los funcionarios, desempleados y jubilados creen que los contenidos en internet deben ser gratis.

La calidad de los contenidos es el principal motivo por el que toda la muestra, independientemente de los ingresos declarados, estaría dispuesta a pagar por contenidos periodísticos o informativos en internet. La imposibilidad de encontrarlos gratis en otros sitios de internet, es la segunda opción para aquellos que declaran rentas bajas, medio-bajas y medio-altas, pero en las rentas superiores a los 50.000 €/año pasa a quinto lugar, por detrás del interés de los contenidos para el trabajo, porque se trata de información muy concreta sobre un tema determinado y sólo accesible mediante pago y, en tercer lugar, por evitar la publicidad.

En este punto es cuando aparecen claramente las variables de calidad, exclusividad, diferenciación y utilidad, coincidiendo con las claves que se sintetizaban fruto de las entrevistas en profundidad realizadas en la fase anterior, mientras otras variables como el precio, la inmediatez en el acceso o la opción de participar en foros y chats de comentarios apenas son valoradas por los usuarios.

\section{Discusión y conclusiones}

La calidad es el principal valor que, tanto los profesionales como los usuarios, destacan por encima de todos los demás, sin diferencias por edad, sexo, nivel educativo o de ingresos, no sólo para elegir contenidos informativos de una fuente concreta en la oferta global de internet, sino además, a la hora de determinar el pago por esos contenidos.

En el caso de los usuarios, un 66,9\% de los encuestados valora la calidad de la información, muy por encima de la segunda opción, que la plataforma elegida sea rápida y práctica. El $48 \%$ además destaca la calidad como la primera variable 


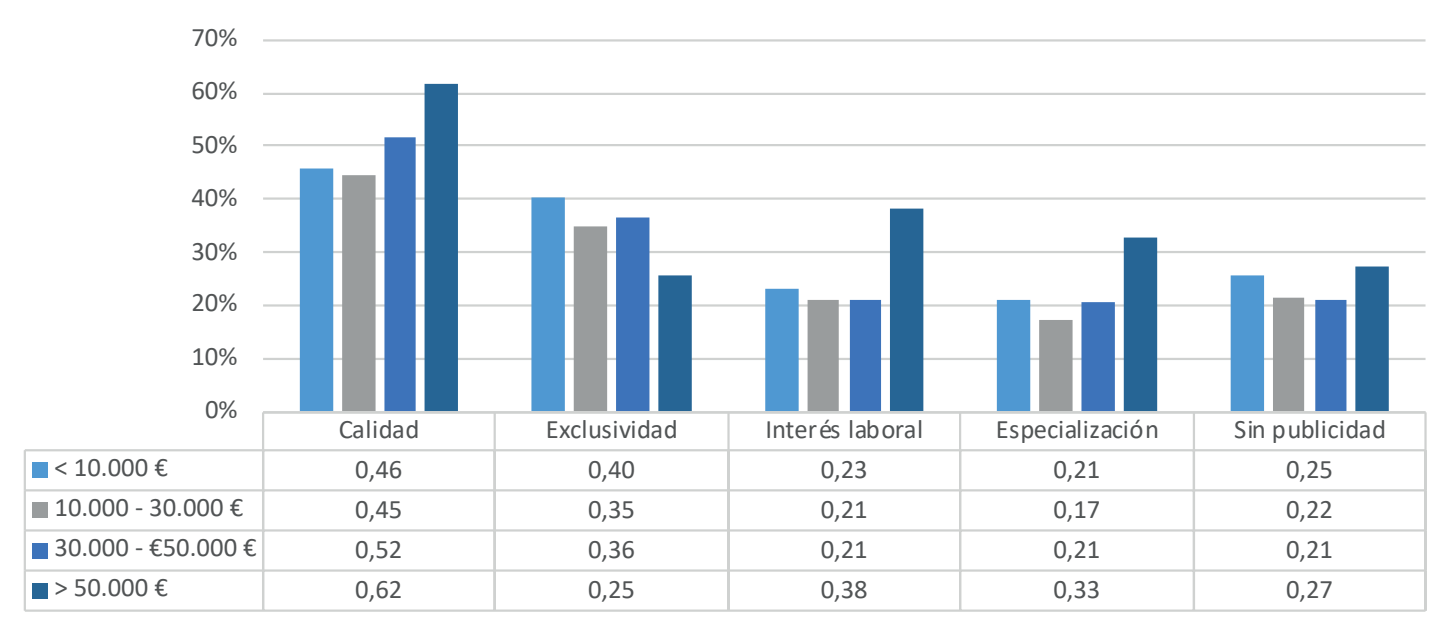

Grafico 6. Principales variables determinantes del pago en función ingresos anuales (respuesta múltiple)

que observaría en una situación de pago por contenidos, seguido a distancia, (un 35,9\%) por la imposibilidad de encontrar esos contenidos de forma gratuita en internet.

La calidad, según la información extraída de las entrevistas en profundidad a los profesionales, se asocia como hemos visto en el modelo propuesto, a valores como la exclusividad, diferenciación, especialización o accesibilidad.

La diferenciación de los demás se valora por parte de los usuarios a través de contenidos exclusivos, tantos reportajes de investigación (47,3\%), informes y documentos explicativos $(46,6 \%)$ o enlaces a otras páginas relacionadas con la información (37\%). En un mundo en el que las exclusivas tal y como las conocíamos, no duran más allá de unos minutos y se vuelven virales a través de las redes sociales, los expertos coinciden al otorgar la cualidad de elemento diferenciador al propio tratamiento de la información y la apuesta por temas propios. No obstante, Rodríguez-Gómez y Sandoval-Martín (2016); Goyanes y Vara-Miguel (2017) ya habían constatado que el interés por estos contenidos no implica la disposición a pagar por ellos. En cualquier caso, no se trata tanto de grandes exclusivas informativas como de ofrecer la información de forma diferenciada, cuidada, con extras y mayores apoyos visuales de todo tipo.

Los expertos apuestan por un contenido diferenciado, original, novedoso, con un tratamiento distinto, con el marchamo de calidad de comentaristas competentes y que sean referentes sociales o culturales y cada vez más dirigido a un segmento de usuario concreto. La diferenciación también se aprovecha de las posibilidades tecnológicas y apuesta por nichos hasta hace poco desdeña-

La calidad es el principal valor que, tanto los profesionales como los usuarios, destacan por encima de todos los demás, sin diferencias por edad, sexo, nivel educativo o de ingresos

dos por los grandes medios, como la hiperproximidad de los medios locales. Los expertos consultados coinciden en que no hay un consumidor general hacia el que lanzar un mensaje masivo como hasta ahora. En la era de internet, aparecen distintos tipos de consumidores que buscan productos diferentes.

Los jóvenes, por el mercado potencial de futuro que suponen, son uno de los targets que más interés despierta para los entrevistados. Además, como hemos podido observar a lo largo de nuestra encuesta, los menores de 24 años marcan diferencias importantes con el resto de la muestra a la hora de consumir información, establecer prioridades o puntos de interés y mostrar disposición al pago. Los datos de la encuesta confirman lo que apuntan los profesionales, que los jóvenes consumen de forma distinta, buscan sensaciones más que contenidos, aceptan una forma de publicidad personal, con una narrativa adaptada y que se descargan cuando quieren. Si los medios son capaces de procesar la información con la que ya cuentan y de crear contenidos adaptados a sus demandas, las posibilidades de establecer un pago por contenidos como ya ha ocurrido en el mundo de la música, los videojuegos o la ficción se pueden convertir en realidad.

Por lo tanto, y como recomendaciones para la gestión podemos concluir que los medios deberán aprovechar las innovaciones técnicas para desarrollar nuevos productos y servicios atractivos, exclusivos y personalizados basados en los datos sobre sus gustos e intereses que los propios usuarios facilitan y por los que estén dispuestos a pagar. Entre las líneas a explorar destaca la identificación de los usuarios con una línea de pensamiento, no necesariamente ideológica, sino también vinculada a aficiones, intereses, proximidad geográfica o ámbito laboral. En cualquier caso, el modelo de negocio sustentado exclusivamente en el pago por contenidos en el caso de la información de actualidad está todavía lejos de ser una realidad. Sólo un modelo mixto, que combine nuevos formatos de publicidad, interesante y personalizado, un muro de pago flexible vinculado a temas exclusivos y especializados, un precio ajustado por un contenido de calidad y el acceso sencillo y en multiplataforma, puede hacer viable a medio y largo plazo un medio de comunicación en internet. 


\section{Bibliografía}

Aparici, Roberto; García-Marín, David (2018). "Prosumidores y emirecs: Análisis de dos teorías enfrentadas". Comunicar, v. 26, n. 55, pp. 71-79.

https://doi.org/10.3916/C55-2018-07

Cabezuelo-Lorenzo, Francisco (2013). “Cinco años de crisis en el mercado de la comunicación (2008-2013)". Historia y comunicación social, v. 18, n. esp. dic., pp. 703-715.

https://doi.org/10.5209/rev_HICS.2013.v18.44358

Campos-Freire, Francisco (coord.) (2010a). El cambio mediático. Zamora: Comunicación Social. ISBN: 9788492860265

Campos-Freire, Francisco (2010b). "Los nuevos modelos de gestión de las empresas mediáticas". Estudios sobre el mensaje periodístico, v. 16, pp. 13-30.

http://revistas.ucm.es/index.php/ESMP/article/view/ESMP1010110013A

Casero-Ripollés, Andreu (2012). "Contenidos periodísticos y nuevos modelos de negocio: evaluación de servicios digitales". El profesional de la información, v. 21, n. 4, pp. 441-446.

https://doi.org/10.3145/epi.2012.jul.02

Castells, Manuel (2009). Comunicación y poder. Madrid: Alianza Editorial. ISBN: 9788420684994

Cea-Esteruelas, María-Nereida (2013). “Economía de los cibermedios: modelo de ingresos y fuentes de financiación". El profesional de la información, v. 22, n. 4, pp. 353-361.

https://doi.org/10.3145/epi.2013.jul.12

Chang, Man-Kit; Cheung, Waiman; Lai, Vincent S. (2005). "Literature derived reference models for the adoption of online shopping". Information \& management, v. 42, n. 4, pp. 543-559.

https://doi.org/10.1016/j.im.2004.02.006

Chen, Zhan; Dubinsky, Alan J. (2003). "A conceptual model of perceived customer value in e-commerce: A preliminary investigation". Psychology \& marketing, v. 4, n. 20, pp. 323-347.

https://doi.org/10.1002/mar.10076

Chyi, Hsiang-Iris (2012). "Paying for what? How much? And why (not)? Predictors of paying intent for multiplatform newspapers". International journal on media management, v. 14, n. 3, pp. 227-250.

https://doi.org/10.1080/14241277.2012.657284

Chyi, Hsiang-Iris; Lasorsa, Dominic L. (2009). "An explorative study on the market relation between online and print newspapers". Journal of media economics, v. 15, n. 2, pp. 91-106.

https://doi.org/10.1207/s15327736me1502_2

Chyi, Hsiang-Iris; Yang, Mengchieh-Jacie (2009). "Is online news an inferior good? Examining the economic nature of online news among users". Journalism \& mass communication quaterly, v. 3, n. 83, pp. 594-612.

https://doi.org/10.1177/107769900908600309

De-Waal, Ester; Schoenbach, Klaus (2010). "News sites position in the mediascape: uses, evaluations and media displacement effects over time". New media \& society, v. 12, n. 3, pp. 477-496.

https://doi.org/10.1177/1461444809341859

Echeverría, Martín; González, Rubén-Arnoldo (2018). “Lógica mediática y comercialismo periodístico. Un análisis longitudinal de encuadres en la cobertura electoral". Communication \& society, v. 31, n. 1, pp. 57-71.

https://www.unav.edu/publicaciones/revistas/index.php/communication-and-society/article/view/35723

Franco-Álvarez, Guillermina (2005). “Los portales periodísticos españoles. El valor añadido de la información de proximidad". Telos. Cuadernos de comunicación e innovación n. 63, pp. 67-69.

https://telos.fundaciontelefonica.com/archivo/numero063/el-valor-anadido-de-la-informacion-de-proximidad/?output=pdf

Gallardo-Olmedo, Fernando (2011). "En la era de los contenidos digitales: nuevos modelos de negocio para la prensa". Telos. Cuadernos de comunicación e innovación, n. 86, pp. 76-86.

http://hdl.handle.net/10486/662225

Gallarza, Martina G.; Gil-Saura, Irene (2006). "Desarrollo de una escala multidimensional para medir el valor percibido de una experiencia de servicio". Revista española de investigación de marketing, v. 2, n. 10, pp. 25-59.

https://www.esic.edu/documentos/revistas/reim/070704_185800_E.pdf

Goyanes, Manuel (2013a). "Conductores de valor de la prensa digital con estrategias de pago por contenidos: análisis del caso del Financial Times, The Times y El Mundo en Orbyt". Palabra clave, v. 16, n. 3, pp. 873-910.

https://doi.org/10.5294/pacla.2013.16.3.8 
Goyanes, Manuel (2013b). "Estrategias y modelos de negocio: aclaración de conceptos y terminología de la prensa en internet". Estudios sobre el mensaje periodístico, v. 19, n. 1, pp. 419-431.

https://doi.org/10.5209/rev_ESMP.2013.v19.n1.42530

Goyanes, Manuel; Vara-Miguel, Alfonso (2017). "Probabilidad de pagar por noticias digitales en España". El profesional de la información, v. 26, n. 3, pp. 488-496.

https://doi.org/10.3145/epi.2017.may.15

Gubbins, Michael (2012). “Digital revolution. Active audiences and fragmented consumption”. En: Lordanova, Dina; Cunnighan, Stuart. Digital disruption: Cinema moves on-line. St. Andrews: St. Andrews Film Studies, pp. 67-100. ISBN: 9780956373076

Gutiérrez-Coba, Liliana (2006). “Análisis de la calidad informativa, primer paso hacia el cambio". Palabra clave, v. 9, n. 1, pp. 30-56.

https://www.redalyc.org/html/649/64900102

Ha, Louisa; Zhang, Xiaoqun (2017). “Are online versions of newspapers inferior goods or public goods? Empirical evidence from US national and local markets". China media research, v. 13, n. 1, pp. 3-19.

https://www.thefreelibrary.com/Are+online+versions+of+newspapers+inferior+goods+or+public+goods\%3F+...-a0490983852

Herbert, Jack; Thurman, Neil (2007). "Paid content strategies for news websites: An empirical study of British newspapers' online business models". Journalism practice, v. 1, n. 2, pp. 208-226.

https://doi.org/10.1080/17512780701275523

Holbrook, Morris B.; Schindler, Robert M. (1994). “Age, sex, and attitude toward the past as predictors of consumers' aesthetic tastes for cultural products". Journal of marketing research, v. 31, n. 3, pp. 412-422.

https://doi.org/10.2307/3152228

Iniesta-Alemán, Isabel; Marta-Lazo, Carmen; Zaro-Becas, María-de-la-Cruz (2018). "La inversión en publicidad, retos para la televisión del siglo XXI". Retos. Revista de ciencias de la administración y economía, v. 8, n. 16, pp. 141-156. https://doi.org/10.17163/ret.n16.2018.10

Jenkins, Henry (2006). Convergence culture: Where old and new media collide. New York: New York University Press. ISBN: 9780814742952

Jenkins, Henry (2008). Convergence culture: la cultura de la convergencia en los medios de comunicación. Barcelona: Paidós. ISBN: 9788449321535

Kerin, Roger A.; Varadarajan, P. Rajan; Peterson, Robert (1992). "First-mover advantaje: A synthesis, conceptual framework and research propositions". Journal of marketing, v. 56, n. 4, pp. 33-52.

https://doi.org/10.2307/1251985

Kotler, Philip (1996). Dirección de mercadotecnia: análisis, planeación, implementación y control. México D.F.: Prentice Hall Hispanoamericana. ISBN: 9789688806760

http://observatoriocultural.udgvirtual.udg.mx/repositorio/handle/123456789/403

Lastra, Ana (2016). “El poder del prosumidor. Identificación de sus necesidades y repercusión en la producción audioviusal transmedia". Icono14, v. 14, n. 1, pp. 71-94.

https://doi.org/10.7195/ri14.v14i1.902

Levine, Rick; Locke, Christopher (2000). El Manifiesto Cluetrain: el ocaso de la empresa. Barcelona: Deusto. ISBN: 978 8423426935

MacNamara, Jim (2010). "Remodelling media: the urgent search for new media business models". Media international Australia, v. 137, n. 1, pp. 20-35.

https://doi.org/10.1177/1329878X1013700104

Marta-Lazo, Carmen; García-Idiakez, Mikel (2014). “El uso profesional de la red social Twitter en la redacción del diario español El país". Palabra clave, v. 2, n. 17, pp. 353-377.

https://doi.org/10.5294/pacla.2014.17.2.4

Marta-Lazo, Carmen; Segura-Anaya, Ana; Martínez-Oliván, Natalia (2017). “Variables determinantes en la disposición al pago por contenidos informativos en internet: perspectiva de los profesionales". Revista latina de comunicación social, n. 72 , pp. 165-185.

https://doi.org/10.4185/RLCS-2017-1159

McLuhan, Marshall; Nevitt, Barrington (1972). Take today: The executive as dropout. New York: Harcourt Brace Jovanovish. ISBN: 9780151878307 
Miguel-De-Bustos, Juan-Carlos; Casado-Del-Río, Miguel-Ángel (2010). “Sobre pago y gratuidad. Momentos de crisis en la industria periodística". Telos. Cuadernos de comunicación e innovación, n. 83, pp. 24-35.

https://telos.fundaciontelefonica.com/archivo/numero083/sobre-pago-y-gratuidad-momentos-de-crisis-en-laindustria-periodistica

Oliver, Richard L.; Swan, John E. (1989a). "Equity and disconfirmation perceptions as influences on merchant and product satisfaction". Journal of customers research, v. 16, n. 3, pp. 372-383.

https://www.jstor.org/stable/2489518

Oliver, Richard L.; Swan, John E. (1989b). "Consumer perceptions of interpersonal equity and satisfaction in transactions: a field survey approach". Journal of marketing, v. 53, n. 2, pp. 21-35.

https://doi.org/10.2307/1251411

Ontsi (2017a). Estudio de uso y actitudes de consumo de contenidos digitales. Observatorio Nacional de las Telecomunicaciones y de la Sociedad de la Información.

https://bit.ly/2PwZRnu

Ontsi (2017b). Informe anual del sector de los contenidos digitales en España 2017. Observatorio Nacional de las Telecomunicaciones y de la Sociedad de la Información.

https://bit.ly/2rCmQFH

Parasuraman, A. Parsu (1997). "Reflections on gaining competitive advantage through customer value". Journal of the Academy of Marketing Science, v. 2, n. 25, pp. 154-161.

https://doi.org/10.1007/BF02894351

Parasuraman, A. Parsu; Grewal, Dhruv (2000). "The impact of technology on the quality-value-loyalty chain: A research agenda". Journal of the Academy of Marketing Science, v. 1, n. 28, pp. 168-174.

https://doi.org/10.1177/0092070300281015

Parra, David; Edo, Concha; Flores, Jesús; García-Alonso, Pedro; Marcos, Juan-Carlos (2008). "Proceso de transformación de los cibermedios: los retos de las empresas periodísticas". Revista latina de comunicación social, n. 63, pp. 63-70. https://mdc.ulpgc.es/cdm/singleitem/collection/rldcs/id/658/rec/6

Pew Research Center (2010). State of the news media 2010.

https://www.pewresearch.org/internet/2010/03/15/state-of-the-news-media-2010

Picard, Robert G. (2004). “Commercialism and newspaper quality”. Newspaper research journal, v. 25, n. 1, pp. 54-65. https://doi.org/10.1177/073953290402500105

PWC (2014). Global entertaiment and media oultlook: 2009-2013. Resumen ejecutivo. Price Waterhouse Coopers. https://www.pwc.com/cl/es/publicaciones/assets/global-entertainment-media.pdf

PWC (2017). Global entertaiment and media oultlook: 2016-2020 España. Price Waterhouse Coopers. https://www.pwc.es/es/entertainment-media-outlook-2016-2020-espana.html

Reig, Ramón (2015). Crisis del sistema, crisis del periodismo: contexto estructural y deseos de cambio. Barcelona: Gedisa. ISBN: 9788497849128

Reuters Institute (2014; 2015; 2016; 2017). Digital news report. Reuters Institute; Oxford University; Center for Internet Studies and Digital Life; Universidad de Navarra.

http://www.digitalnewsreport.org

Rodríguez-Gómez, Eduardo-Francisco; Sandoval-Martín, María-Teresa (2016). “Interés y disposición al pago por investigaciones periodísticas: ¿una solución a la crisis del periodismo?”. Communication \& society, v. 29, n. 1, pp. 1-20. https://www.unav.edu/publicaciones/revistas/index.php/communication-and-society/article/view/35930 https://doi.org/10.15581/003.29.1.sp.1-20

Toffler, Alvin (1981). La tercera ola. México: E. División. ISBN: 9788401370663

Vara-Miguel, Alfonso; Sanjurjo, Elena; Díaz-Espina, Carolina (2012). “Modelos de negocio y estrategia editorial. El caso del wjs.com". Revista de comunicación, n. 11, pp. 1-19.

http://dadun.unav.edu/handle/10171/27752

Victoria-Mas, María; Lacasa-Mas, Iván (2016). “Calidad, credibilidad y valor de marca en las empresas de prensa”. En: Rodríguez-Rodríguez, Jorge-Miguel. Retroperiodismo, o el retorno a los principios de la profesión periodística. Madrid: Sociedad Española de Periodística, pp. 29-42. ISBN: 9788494494123

WAN (2010). "The paid vs. free content debate". Shaping the future of the newspaper. Strategy report, v. 9, n. 2, pp. 1-38. World Association of Newspapers.

http://www.economistiassociati.com/files/erjo_part2_annex_0.pdf 
Wang, Cheng-Lu; Ye, Li-Richard; Zhang, Yue; Nguyen, Dat-Dao (2005). "Suscription to fee-based online services: What makes consumer pay for on line content?". Journal of electronic commerce research, v. 6, n. 4, pp. 304-311.

http://www.jecr.org/node/193

Woodruff, Robert B. (1997). "Marketing in the $21^{\text {st }}$ century customer value: The next source for competitive advantage". Journal of the Academy of Marketing Science, v. 25, n. 256, pp. 139-153.

https://doi.org/10.1177/0092070397253006

Yang, Liu; Ha, Louisa; Wang, Fang; Abuljadail, Mohammad (2015). "Who pays for online content? A media dependency perspective comparing young and older people". International journal on media management, v. 17, n. 4, pp. $277-294$. https://doi.org/10.1080/14241277.2015.1107567

Zeithaml, Valarie A. (1988). "Consumer perceptions of price, quality, and value: A means-end model and synthesis of evidence". The journal of marketing, v. 52, n. 3, pp. 2-22.

https://doi.org/10.2307/1251446

\section{Colección de libros de bolsillo El profesional de la información (Editorial UOC) Últimos títulos publicados}
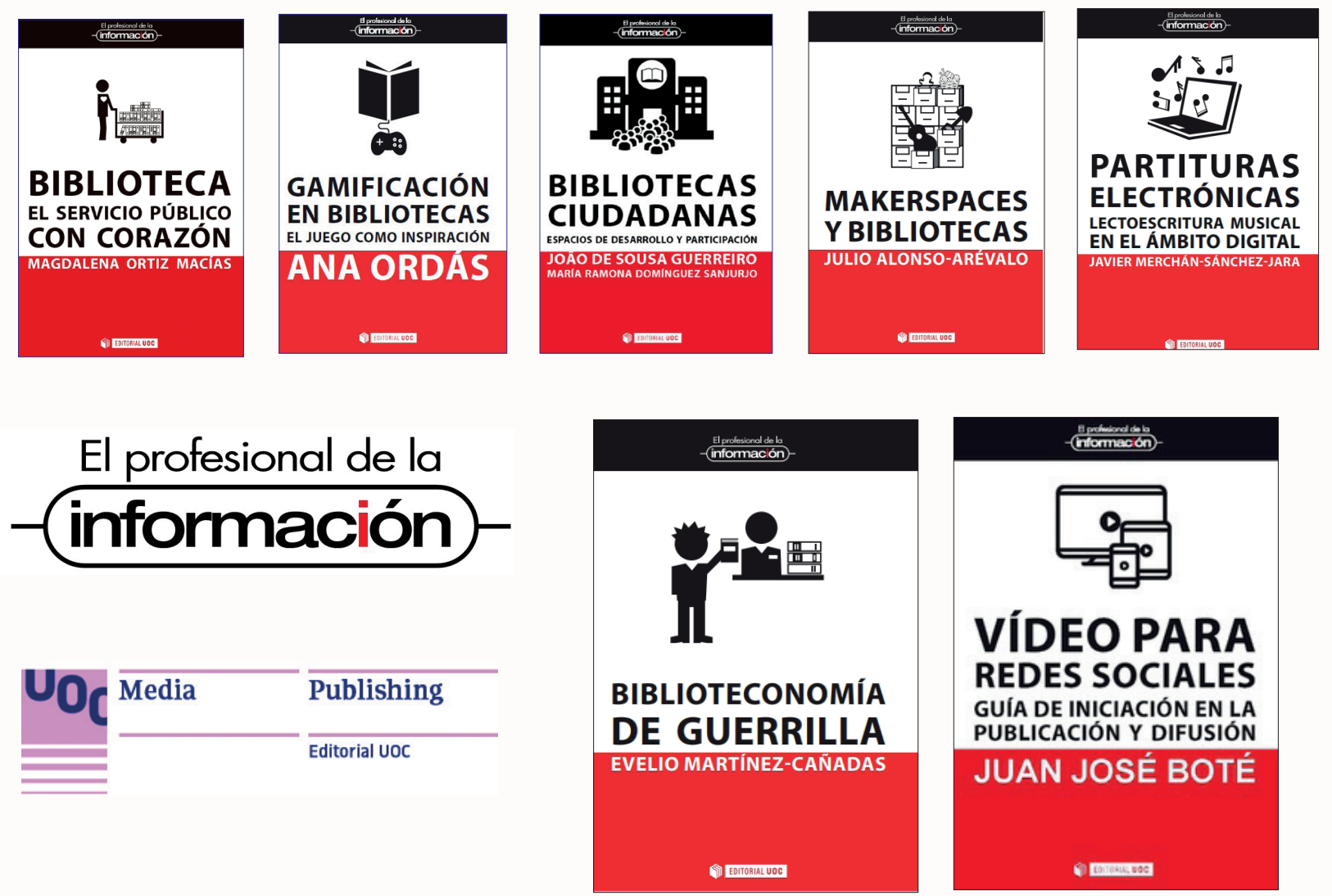

Más información:

http://www.elprofesionaldelainformacion.com/libros.html 